\title{
A spatial analysis of human Schistosoma japonicum infections in Hubei, China, during 2009-2014
}

Hong Zhu' ${ }^{1}$, Shun-Xiang Cai ${ }^{1}$, Jian-Bing Liu' ${ }^{1}, Z u-W u T u^{1}$, Jing Xia ${ }^{1}$, Xiao-Wei Shan ${ }^{1}$, Juan Qiu ${ }^{2}$, Yong Jiang ${ }^{1}$, Ying Xiao', Li Tang ${ }^{1}$ and Xi-Bao Huang ${ }^{1 *}$

\begin{abstract}
Background: The province of Hubei is located in the middle of China, near the middle and lower reaches of the River Yangtze, and is an area where schistosomiasis is endemic. It is challenging to control this disease in this environment, and it would be useful to identify clusters of infection and transmission, as well as their distributions during recent years. Therefore, this study aimed to analyze the spatial distribution of schistosomiasis in Hubei, in order to facilitate the effective control and elimination of this disease.

Methods: We collected schistosomiasis surveillance data from all endemic counties in Hubei during 2009-2014. A geographical information system (ArcGIS, version 10.1) was used to link the counties' geographical data with the epidemiological data, and the spatial scanning method (FleXScan v3.1.2) was used to identify spatial clusters of human infections with Schistosoma japonicum.

Results: In Hubei, patients who exhibited stool test results that were positive for S. japonicum accounted for $>50 \%$ of all cases in China during 2009-2014. However, each endemic county in Hubei exhibited a declining trend in the number of human S. japonicum infections during the study period. The ArcGIS analyses revealed that the middle reaches of the River Yangtze were highly endemic for S. japonicum infections. Spatial scan analyses revealed the following infection clusters: two clusters in ten counties during 2009, two clusters in nine counties during 2010, three clusters in 12 counties during 2011, two clusters in 12 counties during both 2012 and 2013 and two clusters in ten counties during 2014. Most of the cluster regions were located in the lake and marshland regions along the basins of the River Yangtze.

Conclusion: We successfully identified schistosomiasis clusters at the county level in Hubei during 2009-2014, and our results revealed that the clusters were typically located in lake and marshland regions. These data may be useful for controlling and eliminating schistosomiasis in other high-risk areas.
\end{abstract}

Keywords: Spatial analysis, Schistosoma japonicum, Schistosomiasis, Hubei province

\section{Background}

Schistosomiasis is caused by Schistosoma japonicum, and is one of the most serious parasitic diseases in the People's Republic of China [1]. S. japonicum is transmitted by a snail intermediate host, which is a subspecies of Oncomelania hupensis (Gastropoda: Pomatiopsidae). Schistosomiasis has been endemic in 12 Chinese

\footnotetext{
* Correspondence: hxb6407@163.com

${ }^{1}$ Hubei Center for Disease Control and Prevention, Hubei Provincial Academy of Preventive Medicine, Wuhan 430079, China

Full list of author information is available at the end of the article
}

provinces along the River Yangtze, although significant progress has been made in controlling schistosomiasis since the mid-1950s [2]. Since 1985, five provinces and municipalities (Guangdong, Shanghai, Fujian, Guangxi, and Zhejiang) have subsequently fulfilled the criterion for transmission interruption [3]. Thus, schistosomiasis is mainly endemic in lake and marshland regions (located in the provinces of Hubei, Hunan, Jiangxi, Anhui, and Jiangsu) and in hilly and mountainous regions (located in the provinces of Sichuan and Yunnan) [4]. In 2004, a national medium-to-long-term strategic plan was launched 
to accelerate the process of schistosomiasis control throughout China [5]. Furthermore, an integrated strategy that emphasizes infection source control has been in place since 2005 [6], and the schistosomiasis epidemic in China exhibited a dramatic decline after these prevention and control measures were implemented [7].

The province of Hubei is located in the middle of China, near the middle and lower reaches of the River Yangtze (Fig. 1), and is one of the five lake and marshland regions where schistosomiasis remains endemic. $S$. japonicum has existed for over 2100 years in Hubei, and this fact has been confirmed by the presence of $S$. japonicum eggs in a corpse from the Western Han dynasty that was exhumed in 1975 (Jiangling Hsien, Hubei) [8]. In addition to being an endemic area, Hubei has one of the highest rates of schistosomiasis transmission in China [9]. However, the extensive control efforts during recent decades, and especially the integrated and comprehensive control measures that were implemented in 2006, helped Hubei fulfill the criterion for infection control in 2008 (i.e. a human/bovine prevalence of $<5 \%$ in each village) [10]. Therefore, the medium- term goal of schistosomiasis control was successfully achieved in Hubei by the end of 2008 [4]. Subsequent efforts from the central and local governments have helped create a work plan to produce an effective control program. The Hubei government launched the new program ("Provinces and Ministries Union with the Ministry of Health and the Ministry of Agriculture") in 2008, and this program was implemented in 2009 [11]. Between 2009 and 2013, the schistosomiasis control measures included replacing bovines with tractors for agricultural production, rearing domestic animals in pens, preventing domestic animals from roaming in the marshlands, recycling human and livestock excreta for biogas production, controlling Oncomelania species by promoting forestation and moving paddy fields to upland of high-risk areas, improving the water supply, enhancing sanitation and personal hygiene and other routine health-control measures (e.g. praziquantel treatment for humans and bovines, a snail survey and elimination and health education) [12-15]. In 2013, 5 years after this control program was implemented, all endemic counties in Hubei

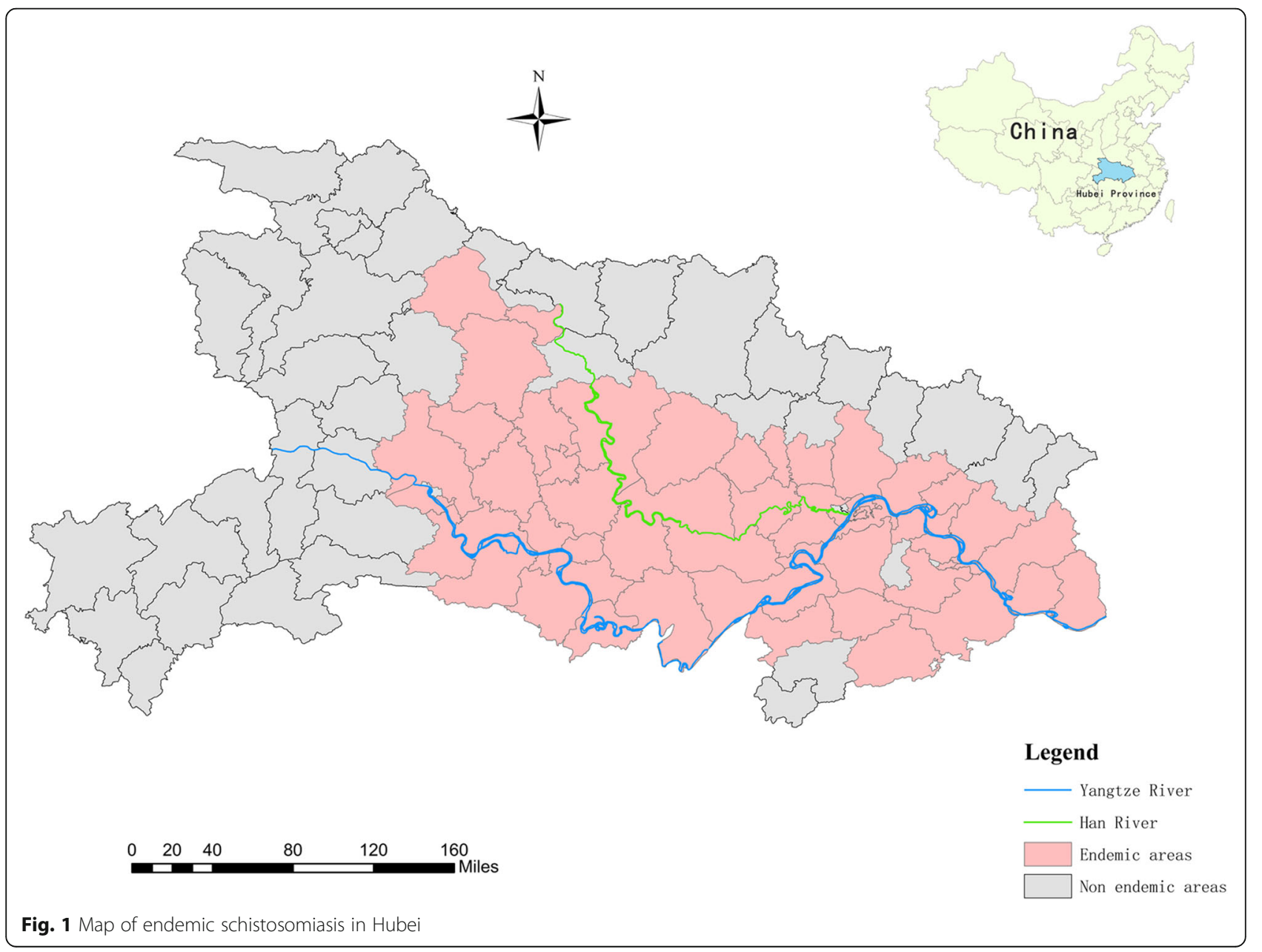


fulfilled the criterion for transmission control (i.e. a human/bovine prevalence of $<1 \%$ in each village and the absence of snail infections). Thus, the longterm goal of schistosomiasis control in Hubei was achieved approximately 2 years ahead of schedule [16].

A few years after achieving schistosomiasis transmission control, the Hubei government attempted to achieve transmission interruption and the elimination of schistosomiasis [17]. However, there are several risk factors that have not been eliminated, and the reemergence of schistosomiasis is possible if efficient control measures are not enforced $[18,19]$. Therefore, it is important to consolidate prevention and treatment efforts, and a new "Control and Elimination of Schistosomiasis" policy (to replace the 2006 policy) was published in 2015 and implemented on January 1, 2016. In this context, the human prevalence of schistosomiasis is an important indicator of transmission interruption and elimination [20], and a stool test is currently the gold standard for detecting and confirming schistosomiasis in humans [10].

The field of spatial analysis in the context of disease research has existed since the 1990s, and this field uses geographical information systems (GIS) to analyze geographical and epidemiological data for specific diseases [21-23]. The recent development of FleXScan software has also allowed researchers to detect clusters of various diseases [24, 25], including the early detection of disease outbreaks [26], cancer [27, 28] and hepatitis A [29]. However, few studies have reported performing a spatial analysis of schistosomiasis clustering using the flexible spatial scan statistic. Furthermore, only one study has performed a spatial analysis of schistosomiasis clustering in Hubei, and there is little information from after the province-wide fulfillment of the national criterion for transmission control in 2013. Therefore, the present study aimed to examine cases of stool test-confirmed $S$. japonicum infection in Hubei, and to use GIS (ArcGIS) and spatial scanning (FleXScan) techniques to analyze the spatial clustering of schistosomiasis at the county level. These data are intended to improve our understanding of the current status of schistosomiasis in the different areas of Hubei, and to provide useful information for the formulation and effective implementation of future control strategies.

\section{Methods}

\section{Study areas}

This study was performed in the province of Hubei (Fig. 1), which is characterized by a relatively wet environment during the summer and a drier environment during the winter. This environment is ideal for the survival of Oncomelania hupensis, which has significantly affected schistosomiasis transmission during recent decades [30]. Furthermore, the residents frequently come into contact with water harbouring infective parasite larvae during their activities of daily living. This study covered 63 endemic counties (Fig. 1) and 5450 endemic villages in Hubei during 2009-2014.

\section{Data collection}

Annual surveys regarding schistosomiasis were performed in each village during the study period; the resulting data were reported to the townships, and final data regarding the numbers of infected individuals were available at the county level. This study targeted residents of each village who were 6-65 years old, and over $90 \%$ of these residents were screened every September to November using the indirect hemagglutination assay $[31,32]$. Stool samples were subsequently collected from over $90 \%$ of the individuals with positive serological results, in order to perform the miracidium-hatching test $[33,34]$. Cases of human S. japonicum infection were defined as patients who exhibited positive results from both the serological and stool tests. We used Microsoft Excel 2007 (Microsoft, Redmond, Washington, USA) to create a database of the surveillance data from the endemic counties during 2009-2014. Data regarding the total number of cases in China were extracted from the annual "Endemic status of schistosomiasis in the People's Republic of China" reports for 2009-2014 [16, 35-39].

\section{Constructing the spatial database}

The spatial database was created by extracting information regarding the cases of $S$. japonicum infection, although these data did not contain coordinate information. Therefore, we extracted each county's coordinates from an electronic map of China $(1: 4,000,000)$ using ArcGIS software (version 10.1) (Esri, Redlands, California, USA), and the geographical data for the endemic counties were merged with the $S$. japonicum infection data. These steps created a spatial database of human $S$. japonicum infections in Hubei that were confirmed using the stool test.

\section{Spatial cluster analysis}

FleXScan software and flexible scan statistics (v 3.1.2) [40] were used to detect irregularly shaped clusters. The data were extracted according to year, and four data files were prepared: a coordinate file, a matrix definition file, a case file and a population file. In the coordinate file, each data line from the schistosomiasis endemic areas in Hubei included the area's name, latitudes and longitudes. In the matrix definition file, which was created using the Hubei provincial atlas, each region was connected to its adjacent regions to establish a spatial matrix of schistosomiasis endemic areas in Hubei. The frequency of disease and the population in each area were included in the case file and population file, respectively. 


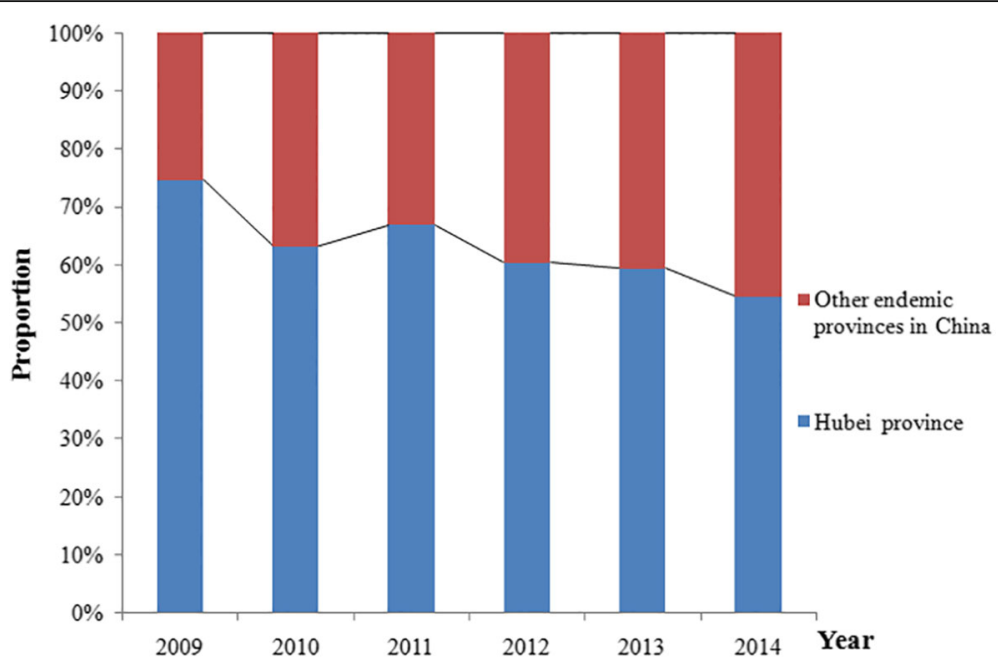

Fig. 2 Changes in the proportions of S. japonicum infections in Hubei and China during 2009-2014

Based on the parameters of the flexible scan statistic, which allow a maximum number of 20 areas per window, we used 15 areas as the limit length of the cluster (default), and a Poisson distribution was used in the probability model. As with the spatial scan statistic, the alternative hypothesis was tested using a log-likelihood ratio (LLR) test and Monte Carlo replications, as well as the expected number of cases and relative risk (RR). Larger LLR values indicate stronger clustering. The RR was calculated for each statistically significant cluster, by comparing the risk inside the cluster with the risk outside the cluster.

For this study, the $P$-value of the test was based on the null distribution of the LLR statistic with a large number of Monte Carlo replications $(n=999)$, using the dataset that was generated under the null hypothesis. Differences were considered statistically significant at a $P$-value of $<0.05$. The flexible spatial scan statistic is also able to locate secondary clusters that do not overlap with the most likely cluster but are still statistically significant. Based on the results of these analyses, we mapped the clustering boundaries using the flexible scan statistics [41].

\section{GIS mapping}

The changes in the numbers of reported cases at the county level in Hubei were plotted using ArcGIS software according to year during 2009-2014. The annual numbers of reported cases at the county level were evaluated to assess the spatial distributions of $S$. japonicum infections. ArcGIS software was also used to visualize the space cluster regions in map.

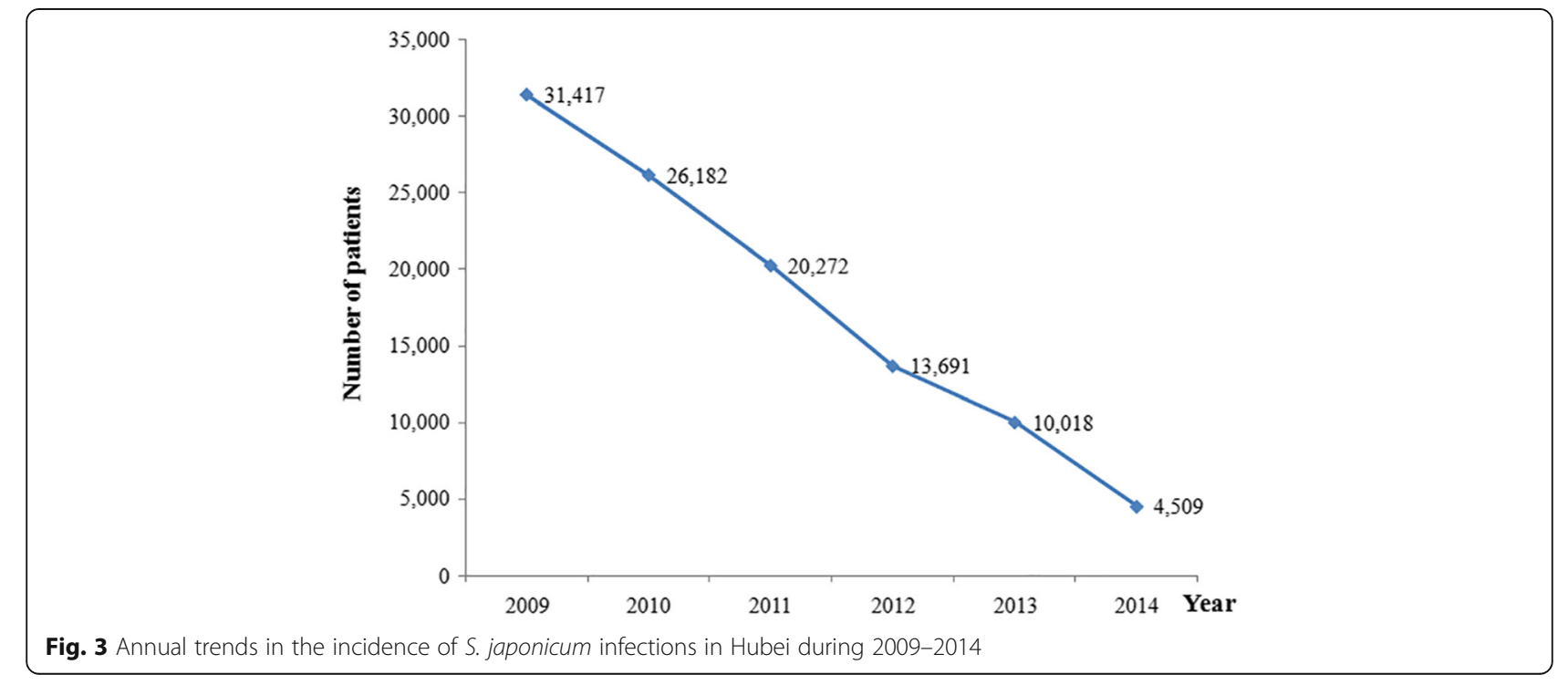


Table 1 The cases of S. japonicum infections in different endemic types of Hubei during 2009-2014

\begin{tabular}{|c|c|c|c|c|c|c|}
\hline \multirow[t]{2}{*}{ Endemic types } & \multicolumn{6}{|l|}{ Year } \\
\hline & 2009 & 2010 & 2011 & 2012 & 2013 & 2014 \\
\hline Infection control & 30,291 & 25,163 & 19,423 & 13,026 & 9620 & 4509 \\
\hline Transmission control & 1122 & 1016 & 849 & 664 & 398 & 0 \\
\hline Transmission interruption & 4 & 3 & 0 & 1 & 0 & 0 \\
\hline Total & 31,417 & 26,182 & 20,272 & 13,691 & 10,018 & 4509 \\
\hline
\end{tabular}

\section{Results}

Endemic status of human S. japonicum infections in Hubei during 2009-2014

When we compared Hubei and the other endemic provinces in China, we found that Hubei accounted for 54.52-74.62 \% of all Chinese cases during the study period (Fig. 2). Although this proportion exhibited a decreasing trend during the study period, Hubei accounted for $>50 \%$ of all Chinese cases in all of the years that we evaluated. The stool test was used to confirm 31,417 cases of human S. japonicum infection in Hubei during 2009, and this number decreased to 4509 cases during 2014, which corresponded to a reduction of $85.65 \%$ (Fig. 3). All cases from 2009 to 2014 could be classified to areas of infection control, transmission control and transmission interruption (Table 1).

\section{Spatial distribution of S. japonicum infections in Hubei}

Each endemic county in Hubei exhibited a declining trend in the incidences of human $S$. japonicum infections during 2009-2014. Counties that were highly endemic were typically located at the middle reaches of the rivers Yangtze and Han (Fig. 4). Among the 63 endemic counties in Hubei, > 500 confirmed cases of human $S$. japonicum infection were observed in 14 counties during 2009, in 11 counties during 2010, in 10 counties during 2011, in 10 counties during 2012, in 7 counties during 2013 and in 3 counties during 2014 (Fig. 5). For a more detailed stratification, the counties were classified as high-risk areas (3000-5000 cases), moderate-risk areas (1500-2999 cases) or low-risk areas (500-1499 cases). Figure 5 shows the changes in the counties' classifications during the study period. We observed 0-3 high-

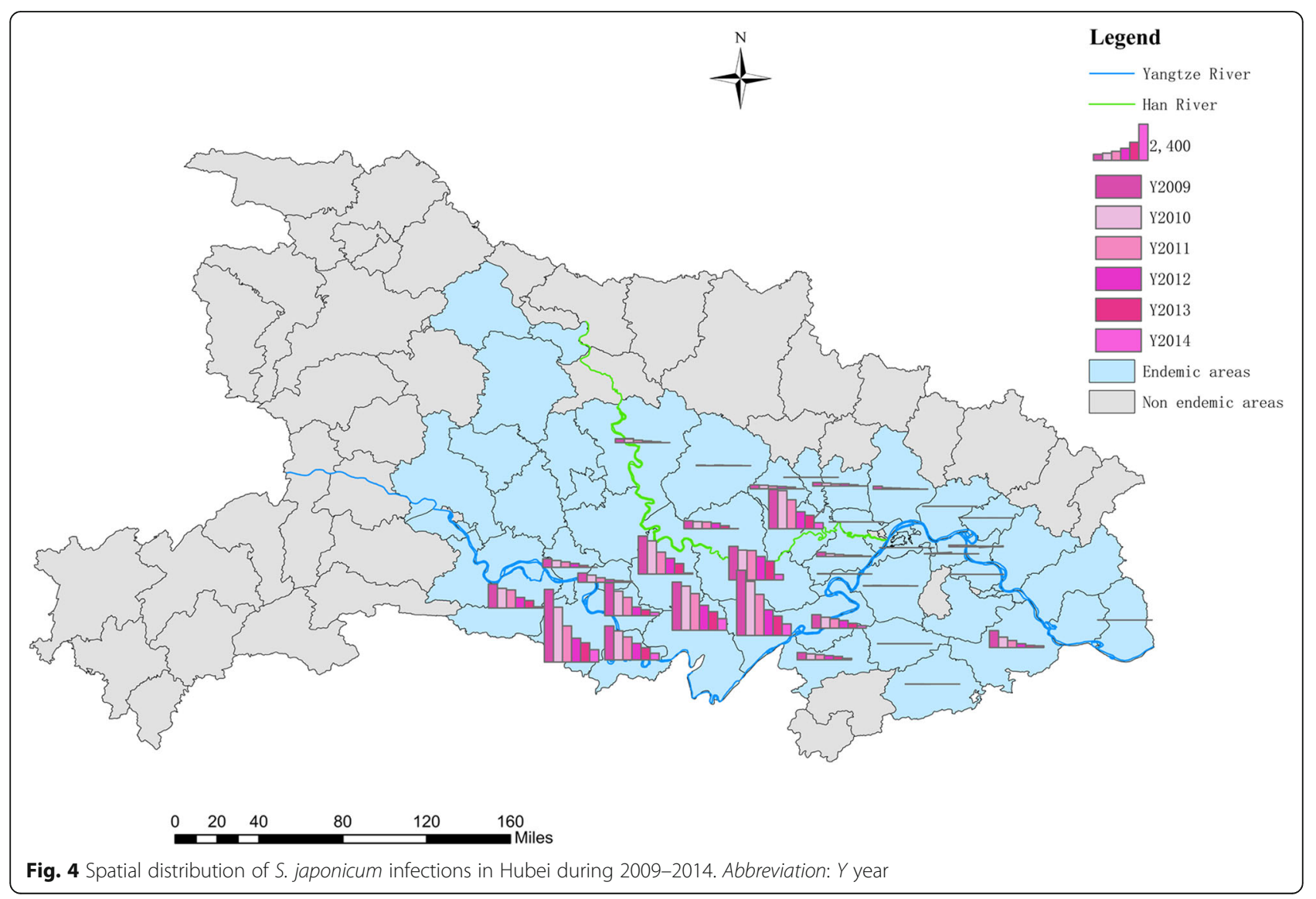




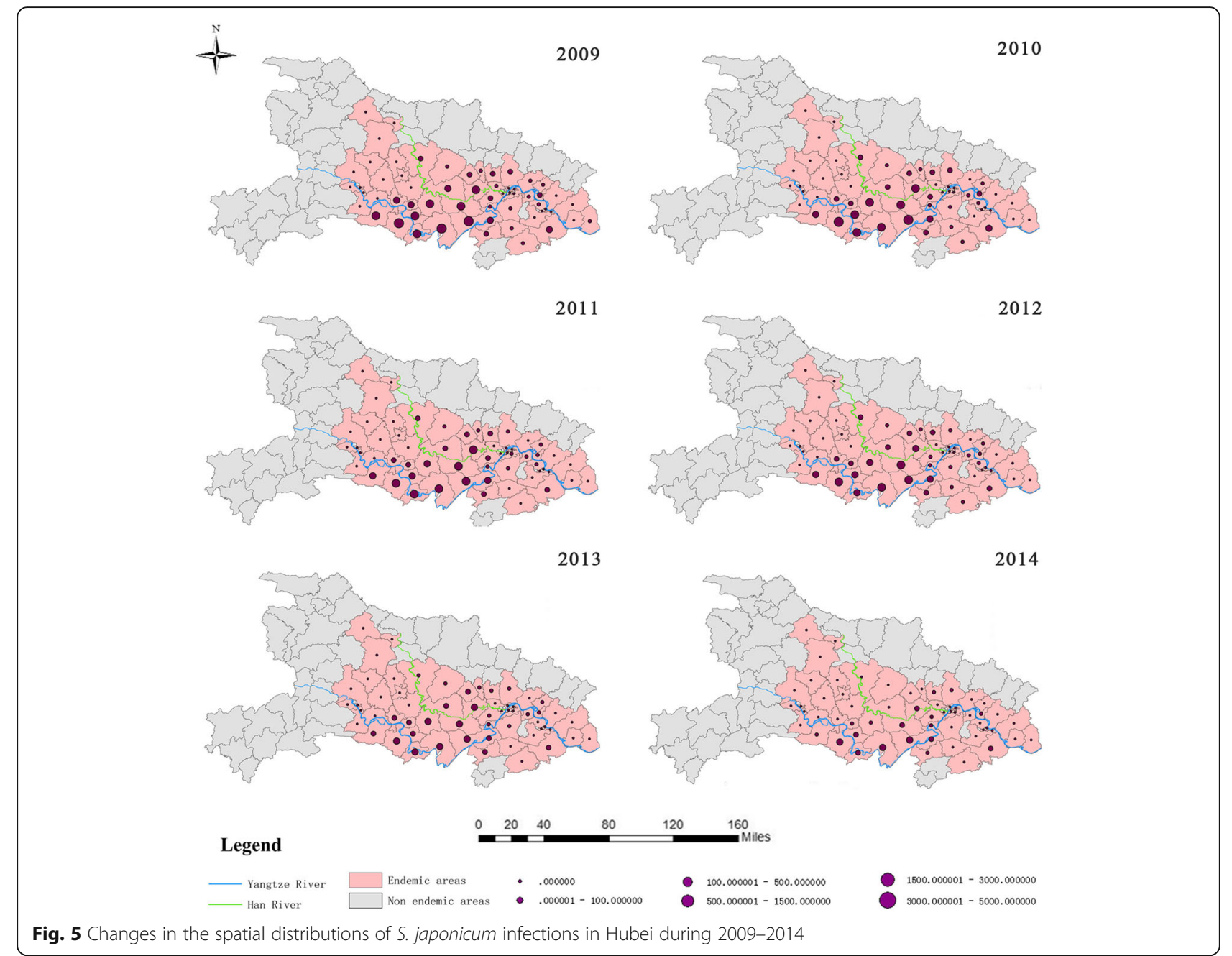

risk areas, 0-6 moderate-risk areas and 3-7 low-risk areas during the study period.

Spatial cluster analysis of S. japonicum infections in Hubei The cluster regions involved 10 counties during 2009, 9 counties during 2010, 12 counties during 2011-2013 and 10 counties during 2014. There was no difference in the 12 cluster regions between 2012 and 2013. Most of the cluster regions were located in lake and marshland regions along the basins of the River Yangtze (Fig. 6). There were three types of significant clusters: 6 most likely clusters, 6 secondary clusters, and 1 tertiary cluster (all $P=0.001$ ). During 2009, the most likely cluster covered 9 counties and exhibited the greatest LLR value during the study period (LLR, 6254; RR, 1.85). The most likely cluster also covered 8 counties in 2009 and 2010, with the exception of Chibi (LLR, 4940; RR, 1.86) and 10 counties in 2011 (LLR, 3465; RR, 1.64). The only tertiary cluster appeared in 2011. In 2014, the most likely cluster covered 9 counties and exhibited the highest $R R$ value during the study (RR, 2.49) (Table 2).

\section{Discussion}

Hubei has been an endemic hotspot for schistosomiasis, which may be related to its lake and marshland regions [42] and other biological and social factors that facilitate the transmission of $S$. japonicum [43]. In the present study, we found that Hubei accounted for $>50 \%$ of all human S. japonicum infections in China during 20092014, which indicates that better control strategies and measures are needed in Hubei. Furthermore, our spatial clustering data may provide useful information for controlling S. japonicum transmission in the lake and marshland regions of China.

During the study period, we observed a declining trend in all measures of $S$. japonicum infections in Hubei (e.g. in relation to all Chinese cases or in the absolute number of confirmed cases). In addition, almost all patients were located in areas that had achieved infection control and transmission control. These changes may be related to the annual surveillance of human infections, livestock infection, and infected snails, as well as the integrated infection control strategy that targeted control 


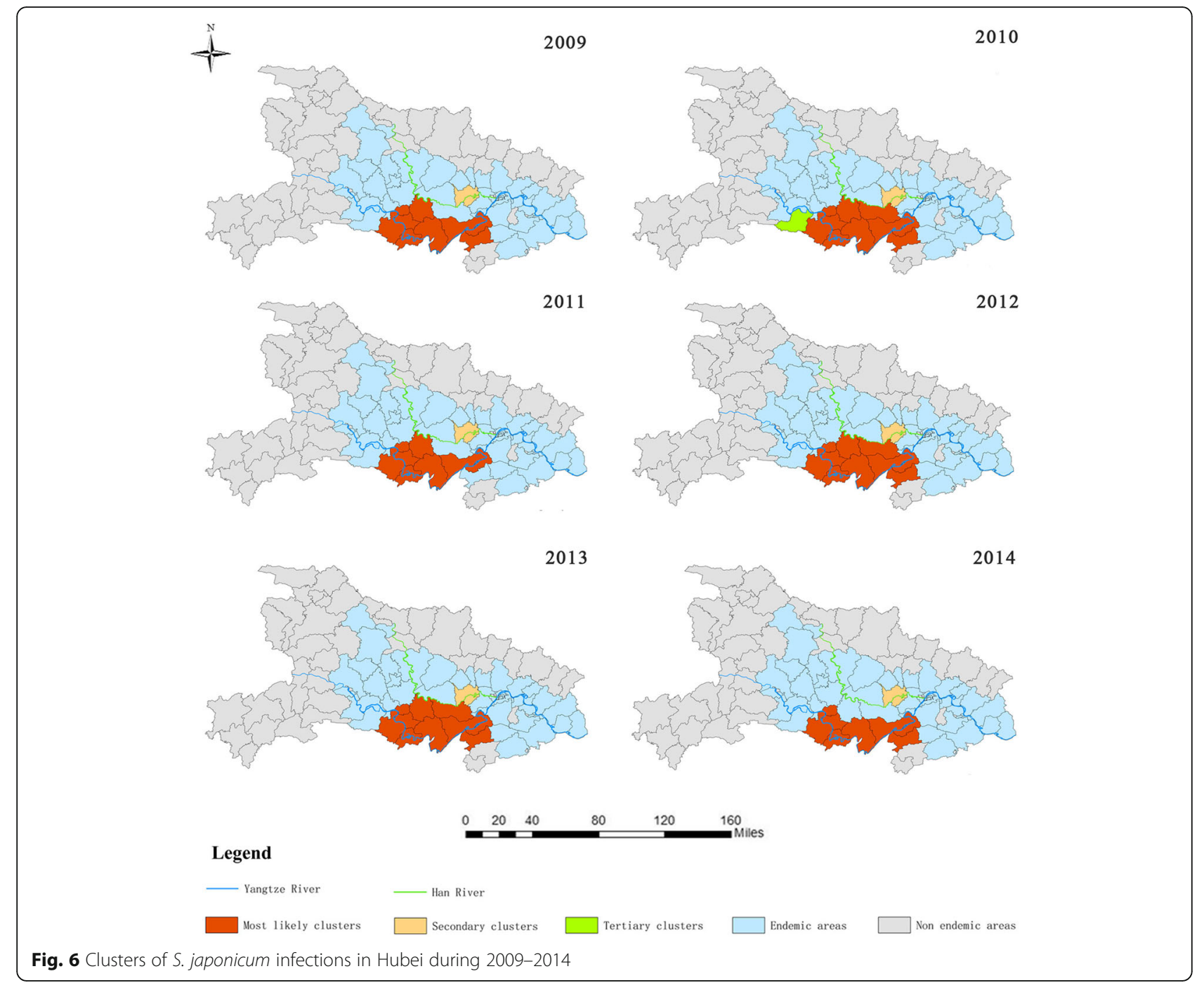

of the sources of infection in Hubei during 2009-2014. For example, the Provinces and Ministries Union program has covered 33 counties since the end of 2008 [44], and the "One Strategy One Village" program has covered 1442 heavily endemic villages since 2012 [45].

Our spatial analyses identified several areas in Hubei where schistosomiasis was endemic, and these areas were typically located along the middle reaches of the River Yangtze. These findings agree with the findings from previous studies that were performed in the provinces of Anhui and Jiangsu [46, 47]. Interestingly, we did not observe any major changes in the spatial distributions over time. This may be related to the fact that agricultural production and social habits in Hubei have not exhibited any major short-term changes, which would indicate that humans and animals still frequently come into contact with infected water, and that the resulting infections are difficult to control. We also used spatial scanning to evaluate clusters of infection during the study period, and our findings were similar to the spatial clustering of acute $S$. japonicum infections in China during 2005-2012 [48] and the high-risk areas that were identified before Hubei achieved schistosomiasis transmission control in 2013 [45]. In addition, although the total number of cases in $2009(31,417)$ decreased to 4509 in 2014 (a reduction of $85.65 \%$ ), we still observed two clusters in 2014. These clusters are likely related to environmental risks in these regions, which contain a mixture of rivers, lakes and marshlands, as well as snails that serve as an infection vector (Oncomelania hupensis). Furthermore, the marshes typically dry up during the winter, and become flooded during the rainy season [49], and the large size of these areas makes it difficult to completely address these issues during a short period of time. Moreover, bovines are especially important to local agricultural production, and these animals can easily become infected in the marshland regions [50,51]. These factors would indicate that residents and animals are still exposed to 
Table 2 The clusters of S. japonicum infections in Hubei during 2009-2014

\begin{tabular}{|c|c|c|c|c|c|c|c|c|}
\hline Year & Types $^{a}$ & $\begin{array}{l}\text { Reported } \\
\text { cases }\end{array}$ & $\begin{array}{l}\text { Expected } \\
\text { cases }\end{array}$ & $\begin{array}{l}\text { Log-likelihood } \\
\text { ratio }\end{array}$ & $\begin{array}{l}\text { Relative } \\
\text { risk }\end{array}$ & $P$-value & $\begin{array}{l}\text { No. of counties } \\
\text { in cluster }\end{array}$ & Areas (County) \\
\hline \multirow[t]{2}{*}{2009} & 1 & 21,224 & 11,443 & 6254 & 1.85 & 0.001 & 9 & $\begin{array}{l}\text { Shashi, Gong'an, Jianli, Jiangling, Shishou, } \\
\text { Honghu, Jiayu, Chibi, Qianjiang }\end{array}$ \\
\hline & 2 & 2601 & 1657 & 244 & 1.57 & 0.001 & 1 & Hanchuan \\
\hline \multirow[t]{2}{*}{2010} & 1 & 17,072 & 9156 & 4940 & 1.86 & 0.001 & 8 & $\begin{array}{l}\text { Shashi, Gong'an, Jianli, Jiangling, Shishou, } \\
\text { Honghu, Jiayu, Qianjiang }\end{array}$ \\
\hline & 2 & 2528 & 1381 & 408 & 1.83 & 0.001 & 1 & Hanchuan \\
\hline \multirow[t]{3}{*}{2011} & 1 & 14,938 & 9096 & 3465 & 1.64 & 0.001 & 10 & $\begin{array}{l}\text { Shashi, Gong'an, Jianli, Jiangling, Shishou, } \\
\text { Honghu, Jiayu, Chibi, Xiantao, Qianjiang }\end{array}$ \\
\hline & 2 & 1921 & 1069 & 293 & 1.8 & 0.001 & 1 & Hanchuan \\
\hline & 3 & 1189 & 1003 & 17 & 1.19 & 0.001 & 1 & Songzi \\
\hline \multirow[t]{2}{*}{2012} & 1 & 10,356 & 6243 & 2562 & 1.66 & 0.001 & 11 & $\begin{array}{l}\text { Shashi, Jingzhou Development Zone, Gong'an, } \\
\text { Jianli, Jiangling, Shishou, Honghu, Jiayu, Chibi, } \\
\text { Xiantao, Qianjiang }\end{array}$ \\
\hline & 2 & 1122 & 722 & 101 & 1.55 & 0.001 & 1 & Hanchuan \\
\hline \multirow[t]{2}{*}{2013} & 1 & 7737 & 4568 & 2090 & 1.69 & 0.001 & 11 & $\begin{array}{l}\text { Shashi, Jingzhou Development Zone, Gong'an, } \\
\text { Jianli, Jiangling, Shishou, Honghu, Jiayu, Chibi, } \\
\text { Xiantao, Qianjiang }\end{array}$ \\
\hline & 2 & 887 & 528 & 108 & 1.68 & 0.001 & 1 & Hanchuan \\
\hline \multirow[t]{2}{*}{2014} & 1 & 3445 & 1383 & 1998 & 2.49 & 0.001 & 9 & $\begin{array}{l}\text { Shashi, Jingzhou Development Zone, Gong'an, } \\
\text { Jianli, Jiangling, Shishou, Honghu, Jiayu, Chibi }\end{array}$ \\
\hline & 2 & 402 & 238 & 50 & 1.69 & 0.001 & 1 & Hanchuan \\
\hline
\end{tabular}

${ }^{\mathrm{a}} 1$, most likely clusters; 2 , secondary clusters; 3 , tertiary clusters

infection, because of their proximity to the River Yangtze and its branches. Therefore, control efforts should likely target these cluster areas, and new techniques and methods should be used to address the epidemic in these areas.

The present study includes several strengths. First, our analysis is based on absolute quantities, rather than the relative indicators of infection that have been used in previous studies (e.g. the infection rate or positive rate). Secondly, our spatial analysis using the flexible spatial scan statistic allowed us to identify infection clusters, which will be useful for directing future efforts to control schistosomiasis. However, this study also involves several limitations. First, our analyses were limited by the absence of detailed demographic data (e.g. sex, age and occupation), which precludes any adjustment for potentially confounding factors. Secondly, we only evaluated the incidences of $S$. japonicum infection at the county level, and additional studies are needed to provide higher-resolution spatial distributions (e.g. village or individual levels).

\section{Conclusion}

In the present study, we identified county-based clusters of human S. japonicum infections in Hubei during 20092014, using positive stool test results and FleXScan software. These results indicate that control efforts should be focused on the middle reaches of the River Yangtze and its connecting branches. Given the importance of accurate epidemiological data regarding schistosomiasis, our results suggest that it may be useful to create a database of patients who exhibit positive stool test results, in order to facilitate dynamic monitoring of schistosomiasis.

Abbreviations

GIS: Geographical Information Systems; LLR: Log-likelihood ratio; RR: Relative risk

\section{Acknowledgments}

The authors thank the staff at the local Center for Disease Control \& Prevention and the Schistosomiasis Institute of Prevention and Control for their collaboration and for providing the field data.

\section{Funding}

Not applicable.

\section{Availability of data and material}

The dataset supporting the conclusions of this article is included within the article and its additional files.

\section{Authors' contributions}

$H Z, X H, S C$, and $J L$ conceived and designed the study. $H Z, X H, S C, J L, Z T, X S$, and LT performed the study. $H Z$ and $Y X$ collected the data. HZ, JX, XS, and $J Q$ analyzed the data. $\mathrm{HZ}$ and $\mathrm{XH}$ wrote the manuscript. $Y J$ synthesized the figures. All authors read and approved the final manuscript.

\section{Competing interests}

The authors declare that they have no competing interests.

Consent for publication

Not applicable. 


\section{Ethics approval and consent to participate}

The study's protocol was approved by the Ethics Review Committee of the Hubei Provincial Center for Disease Control and Prevention, Wuhan, China. All participants provided written informed consent for the testing and analysis of their data, and were allowed to withdraw from the study at any time. All participants who tested positive for S. japonicum were treated using a single oral dose of praziquantel $(40 \mathrm{mg} / \mathrm{kg}$, the maximum dose was based on a weight of $60 \mathrm{~kg}$ ), in accordance with the World Health Organization's recommendations.

\section{Author details}

${ }^{1}$ Hubei Center for Disease Control and Prevention, Hubei Provincial Academy of Preventive Medicine, Wuhan 430079, China. ${ }^{2}$ Key Laboratory for Environment and Disaster Monitoring and Evaluation, Hubei, Institute of Geodesy and Geophysics, Chinese Academy of Sciences, 430077 Wuhan, China

Received: 23 February 2016 Accepted: 23 September 2016 Published online: 04 October 2016

\section{References}

1. Zhou XN, Bergquist R, Leonardo L, Guo JG, Yang K, Sudomo M, et al. Schistosomiasis japonica: control and research needs. Adv Parasitol. 2010;72:145-72

2. Utzinger J, Zhou XN, Chen MG, Bergquist R. Conquering schistosomiasis in China: the long march. Acta Trop. 2005;96:69-96.

3. Zhou XN, Wang LY, Chen MG, Wu XH, Jiang QW, Chen XY, et al. The public health significance and control of schistosomiasis in China-then and now. Acta Trop. 2005;96:97-105.

4. Collins C, Xu J, Tang SL. Schistosomiasis control and the health system in P.R. China. Infect Dis Poverty. 2012;1:1-8.

5. Zhou YB, Liang S, Chen GX, Rea C, He ZG, Zhang ZJ, et al. An integrated strategy for transmission control of Schistosoma japonicum in a marshland area of China: findings from a five-year longitudinal survey and mathematical modeling. Am J Trop Med Hyg. 2011;85:83-8.

6. Wang LD, Chen HG, Guo JG, Zeng XJ, Hong XL, Xiong JJ, et al. A strategy to control transmission of Schistosoma japonicum in China. N Engl J Med. 2009;360:121-8.

7. Wang LD, Guo JG, Wu XH, Chen HG, Wang TP, Zhu SP, et al. China's new strategy to block Schistosoma japonicum transmission: experiences and impact beyond schistosomiasis. Trop Med Int Health. 2009;14:1475-83.

8. Zhou DR, Li YS, Yang XM. Schistosomiasis control in China. World Health Forum. 1994;15:387-9.

9. Wu XH, Zhang SQ, Xu XJ, Huang YX, Steinmann P, Utzinger J, et al. Effect of floods on the transmission of schistosomiasis in the Yangtze River valley, People's Republic of China. Parasitol Int. 2008:57:271-6.

10. Zhou XN, Xu J, Chen HG, Wang TP, Huang XB, Lin DD, et al. Tools to support policy decisions related to treatment strategies and surveillance of schistosomiasis japonica towards elimination. PLoS Negl Trop Dis. 2011;5: e1408.

11. Xu J, Zhang XF, Gao J, Huang XB, Zhang $L$, Li SZ, et al. Assessment on the effect of joint effort for schistosomiasis control in Hubei province. Chin J Parasitol Parasit Dis. 2014;32:180-5.

12. Zhu H, Huang XB, Cai SX, Tu ZW, Chen YY, Li G, et al. Evaluation of comprehensive measures to control schistosomiasis primarily through infection source control in Hubei Province. Journal of Pathogen Biology. 2011;6:908-11.

13. Zhu H, Chen DE, Huang XB, Cai SX, Tu ZW, Chen YY, et al. Cost-effectiveness of comprehensive measures with emphasis on infection source control for schistosomiasis in marshland endemic regions. J Trop Med. 2010:10:982-5.

14. Zhu H, Cai SX, Huang XB, Tu ZW, Cao MM, Dai LF, et al. Preliminary effect of comprehensive measures with emphasis on infectious source control for schistosomiasis control in Hubei Province. Chin J Schisto Control. 2009;21:267-71

15. Li SZ, Qian YJ, Yang K, Wang Q, Zhang HM, Liu J, et al. Successful outcome of an integrated strategy for the reduction of schistosomiasis transmission in an endemically complex area. Geospat Health. 2012;6:215-20.

16. Lei ZL, Zheng H, Zhang LJ, Zhu R, Xu ZM, Xu J, et al. Endemic status of schistosomiasis in People's Republic of China in 2013. Chin J Schisto Control. 2014;26:591-7.
17. Zhou XN, Bergquist $R$, Tanner M. Elimination of tropical disease through surveillance and response. Infect Dis Poverty. 2013:2:1-5.

18. Dang H, Xu J, Li SZ, Cao ZG, Huang YX, Wu CG, et al. Monitoring the transmission of Schistosoma japonicum in potential risk regions of China 2008-2012. Int J Environ Res Public Health. 2014;11:2278-87.

19. Chen YY, Liu JB, Huang XB, Cai SX, Su ZM, Zhong R, et al. New integrated strategy emphasizing infection source control to curb Schistosomiasis japonica in a marshland area of Hubei province, China: findings from an eight-year longitudinal survey. Plos One. 2014;9:e89779.

20. General Administration of Quality Supervision, Inspection and Quarantine of the People's Republic of China (AQSIQ), Standardization Administration of the People's Republic of China (SAC). The national standard of the People's Republic of China, Control and elimination of schistosomiasis (GB 15976-2015). 2015. http://cspd.ipd.org.cn/Article/bzzd/bzfb/201507/1084.html. Accessed 10 Sep 2015.

21. French $N$, Barrigas $M$, Brown $P$, Ribiero $P$, Williams $N$, Leatherbarrow $H$, et al. Spatial epidemiology and natural population structure of Campylobacter jejuni colonizing a farmland ecosystem. Environ Microbiol. 2005;7:1116-26.

22. Graham AJ, Atkinson PM, Danson FM. Spatial analysis for epidemiology. Acta Trop. 2004;91:219-25

23. Elliott $\mathrm{P}$, Wartenberg D. Spatial epidemiology: current approaches and future challenges. Environ Health Perspect. 2004;112:998-1006.

24. Tango T, Takahashi K. A flexibly shaped spatial scan statistic for detecting clusters. Int J Health Geogr. 2005;4:11

25. Tango T, Takahashi K. A flexible spatial scan statistic with a restricted likelihood ratio for detecting disease clusters. Stat Med. 2012;31:4207-18.

26. Takahashi K, Kulldorff M, Tango T, Yih K. A flexibly shaped space-time scan statistic for disease outbreak detection and monitoring. Int J Health Geogr. 2008;7:14.

27. Ramis R, Gómez-Barroso D, López-Abente G. Cluster detection of diseases in heterogeneous populations: an alternative to scan methods. Geospat Health. 2014:8:517-26.

28. lyengar VS. Space-time clusters with flexible shapes. MMWR Suppl. 2005:54:71-6.

29. Wang XW, Zhao SW, Liu ZT, Yu SY, Zheng GQ, Mei ZC, et al. Compare the application of flexible spatial statistics and kulldorff scanning window statistics to spatial aggregation of hepatitis A. Chin J Prev Med. 2014:48:1063-7.

30. Zhou XN, Bergquist R, Leonardo L, Yang GJ, Yang K, Sudomo M, et al. Schistosomiasis japonica: control and research needs. Adv Parasitol. 2010;72:145-78

31. Zhu YC. Immunodiagnosis and its role in schistosomiasis control in China: a review. Acta Trop. 2005;96:130-6.

32. Cai YC, Xu JF, Steinmann P, Chen SH, Chu YH, Tian LG, et al. Field comparison of circulating antibody assays versus circulating antigen assays for the detection of schistosomiasis japonica in endemic areas of China. Parasit Vectors. 2014:7:138

33. Zhu HQ, Xu J, Zhu R, Cao CL, Bao ZP, Yu Q, et al. Comparison of the miracidium hatching test and modified Kato-Katz method for detecting Schistosoma japonicum in low prevalence areas of China. Southeast Asian J Trop Med Public Health. 2014:45:20-5.

34. Ross AG, Sleigh AC, Li YS, Davis GM, Williams GM, Jiang Z, et al. Schistosomiasis in the People's Republic of China: prospects and challenges for the 21st century. Clin Microbiol Rev. 2001;14:270-95.

35. Hao Y, Zheng H, Zhu R, Guo JG, Wang LY, Chen Z, et al. Schistosomiasis situation in People's Republic of China in 2009. Chin J Schisto Control. 2010;22:521-7.

36. Lei ZL, Zheng H, Zhang LJ, Zhu R, Guo JG, Li SZ, et al. Schistosomiasis status in People's Republic of China in 2010. Chin J Schisto Control. 2011:23:599-604.

37. Zheng H, Zhang LJ, Zhu R, Xu J, Li SZ, Guo JG, et al. Schistosomiasis situation in People's Republic of China in 2011. Chin J Schisto Control. 2012;24:621-6.

38. Li SZ, Zheng H, Gao J, Zhang $\sqcup$, Zhu R, Xu J, et al. Schistosomiasis status in People's Republic of China in 2012. Chin J Schisto Control. 2013;25:557-63.

39. Lei ZL, Zhang LJ, Xu ZM, Dang H, Xu J, Lv S, et al. Endemic status of schistosomiasis in People's Republic of China in 2014. Chin J Schisto Control. 2015:27:563-9.

40. Takahashi K, Yokoyama T, Tango T. FleXScan v3.1.2: Software for the Flexible Scan Statistic. 2013. https://sites.google.com/site/flexscansoftware/download_e. Accessed 15 June 2016 
41. Takahashi K, Yokoyama T, Tango T. FleXScan User Guide for version 3.1. Department of Technology Assessment and Biostatistics National Institute of Public Health. 2010. http://www.niph.go.jp/soshiki/gijutsu/index_e.html. Accessed 15 June 2016.

42. Xia J, Yuan Y, Xu XJ, Wei FH, Li GL, Liu M, et al. Evaluating the effect of a novel molluscicide in the endemic schistosomiasis japonica area of China. Int J Environ Res Public Health. 2014;11:10406-18.

43. Chen $Y Y$, Huang $X B$, Xiao $Y$, Jiang $Y$, Shan XW, Zhang J, et al. Spatial analysis of schistosomiasis in Hubei Province, China: a GIS-based analysis of schistosomiasis from 2009 to 2013. PLoS One. 2015;10:e0118362.

44. Zhu H, Cai SX, Liu JB, Liao HY, Gong XG, Xu XJ, et al. Assessment report on objectives and tasks of schistosomiasis control in Hubei Province (2012). J Pub Health and Prev Med. 2013;24:46-50.

45. Zhu H, Liu JB, Cai SX, Huang XB, Xiao Y, Tu ZW, et al. Compliance risk analysis of schistosomiasis control of the disease controlling areas in Hubei province. J Trop Med. 2014;14:1086-9.

46. Gao FH, Abe EM, Li SZ, Zhang L, He JC, Zhang SQ, et al. Fine scale spatialtemporal cluster analysis for the infection risk of schistosomiasis japonica using space-time scan statistics. Parasit Vectors. 2014;7:578.

47. Yang K, Li W, Sun LP, Huang YX, Zhang JF, Wu F, et al. Spatio-temporal analysis to identify determinants of Oncomelania hupensis infection with Schistosoma japonicum in Jiangsu province. China Parasit Vectors. 2013;6:138.

48. Li SZ, Zheng H, Abe EM, Yang K, Bergquist R, Qian YJ, et al. Reduction patterns of acute schistosomiasis in the People's Republic of China. PLoS Negl Trop Dis. 2014:8:e2849.

49. Hu Y, Zhang ZJ, Chen Y, Wang ZL, Gao J, Tao B, et al. Spatial pattern of schistosomiasis in Xingzi, Jiangxi Province, China: effects of environmental factors. Parasit Vectors. 2013;6:214

50. Gray DJ, Williams GM, Li YS, McManus DP. Transmission dynamics of Schistosoma japonicum in the lakes and marshlands of China. PLoS One. 2008;: $:$ e4058.

51. Zhou YB, Liang S, Jiang QW. Factors impacting on progress towards elimination of transmission of schistosomiasis japonica in China. Parasit Vectors. 2012;5:275.

\section{Submit your next manuscript to BioMed Central and we will help you at every step:}

- We accept pre-submission inquiries

- Our selector tool helps you to find the most relevant journal

- We provide round the clock customer support

- Convenient online submission

- Thorough peer review

- Inclusion in PubMed and all major indexing services

- Maximum visibility for your research

Submit your manuscript at www.biomedcentral.com/submit

) Biomed Central 